\title{
FACTORES DE DUCTILIDAD Y SOBRERRESISTENCIA EN MARCOS DE ACERO CON CONTRAVENTEO CHEVRÓN
}

\author{
Edgar Tapia Hernández ${ }^{(1)}$ y Arturo Tena Colunga ${ }^{(2)}$
}

\begin{abstract}
RESUMEN
En este artículo se presentan los resultados de la valoración de los factores de modificación de carga obtenidos de análisis estáticos no lineales ante carga monótona creciente de 13 edificios regulares de marcos dúctiles de acero con contraventeo concéntrico tipo chevrón. Los modelos con alturas de cuatro a 16 niveles, se diseñaron con el máximo factor de comportamiento sísmico en el Reglamento para el Distrito Federal $(\mathrm{Q}=3)$, variando la aportación al cortante lateral resistente entre las columnas del marco y el sistema de contravientos. Se proponen ecuaciones para obtener factores más realistas de reducción por ductilidad y sobrerresistencia.
\end{abstract}

Palabras clave: marcos dúctiles contraventeados, contraventeo chevrón, diseño por capacidad, distorsiones de diseño, factor de reducción por sobrerresistencia, desempeño estructural.

\begin{abstract}
Results related to the assessment of force modification factors obtained with pushover analyses of 13 regular steel buildings structured with ductile moment - resisting concentrically braced frames with chevron configuration are summarized in this paper. Four-story to 16-story models were designed for the maximum seismic response modification factor $(Q=3)$ allowed in Mexico's Federal District Code. Different balances between the story shear resisted by the columns with respect to the one resisted by the bracing system were considered. Improved equations were obtained for a more realistic assessment for the ductility and overstrength force modification factors.
\end{abstract}

Keywords: ductile braced frames, chevron bracing, capacity design, design drifts, overstrength factor, structural performance.

\section{INTRODUCCIÓN}

A pesar que algunos reglamentos reconocen de tiempo atrás la importancia de considerar la ductilidad $(\mu)$ en el diseño sísmico, sólo las versiones más recientes consideran adicionalmente de manera directa la influencia inherente de la sobrerresistencia en los sistemas estructurales ( $R$ en los reglamentos mexicanos, $\Omega$ en los reglamentos de Estados Unidos).

Artículo recibido el 30 de septiembre de 2010 y aprobado para su publicación el 6 de junio de 2011.

(1) Candidato Doctoral, Posgrado en Ingeniería Estructural, División de Ciencias Básicas e Ingeniería ,Universidad Autónoma Metropolitana Azcapotzalco, Av. San Pablo 180, Col. Reynosa Tamaulipas, 02200 México, DF, e-mail: etapiah@ $\underline{\text { hotmail.com }}$

(2) Profesor, Departamento de Materiales, Universidad Autónoma Metropolitana Azcapotzalco, Av. San Pablo 180, Col. Reynosa Tamaulipas, 02200 México, DF, e-mail: $\underline{\text { atc@correo.azc.uam.mx }}$ 
En estudios recientes de edificios estructurados con marcos de acero contraventeados (Elghazouli 2003, MacRae et al. 2004, Tapia 2005, Lacerte y Tremblay 2006, Izvernari 2007, Tapia y Tena 2009, Fell et al. 2010), se han demostrado que aplicando la metodología propuesta en reglamentos modernos no necesariamente se obtiene, en todos los casos, resultados congruentes con las premisas de diseño, sobre todo si se abusa del uso de las ayudas de diseño del software comercial. En estos estudios de referencia se muestra que estructuras diseñadas bajo la hipótesis que su comportamiento es dúctil pudieran obtenerse mecanismos de colapso mixtos, con considerables reservas de sobrerresistencia, mucho mayores a las supuestas en su diseño.

Conforme al Reglamento de Construcciones para el Distrito Federal, un marco con contravientos debe analizarse considerando la aportación al cortante resistente del marco y el sistema de contravientos (figura 1), siendo que todas las columnas del marco deben ser capaces de soportar al menos el $50 \%$ del cortante lateral para ser considerado marco dúctil. En estas premisas se supone que siguiendo estas recomendaciones de diseño y detallado de elemento, se obtendrán mecanismos de colapso columna fuerte - viga débil - contraviento más débil.

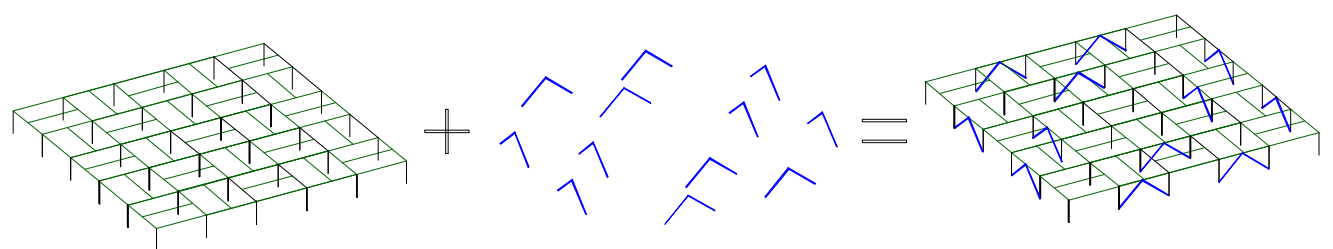

Figura 1. Distribución de los sistemas que conforman el entrepiso contraventeado

Esta investigación presenta un estudio paramétrico de marcos dúctiles de acero con contraventeo concéntrico tipo chevrón, considerando distintas alturas y balances de resistencia entre la aportación de las columnas del marco y el sistema de contraviento.

\section{DESCRIPCIÓN DE LOS MODELOS}

El estudio se concentra en las ductilidades y sobrerresistencias obtenidas de análisis no lineales ante carga estática monótona creciente de trece edificios regulares estructurados con marcos dúctiles de acero con contraventeo concéntrico de 4, 8, 12 y 16 niveles (figura 2).

a)

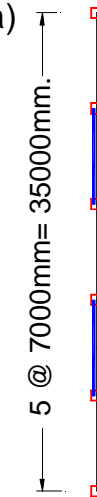

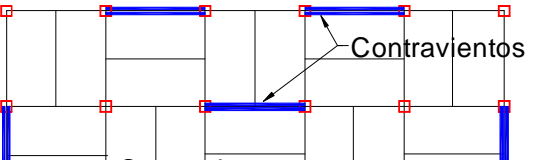

Contravientos
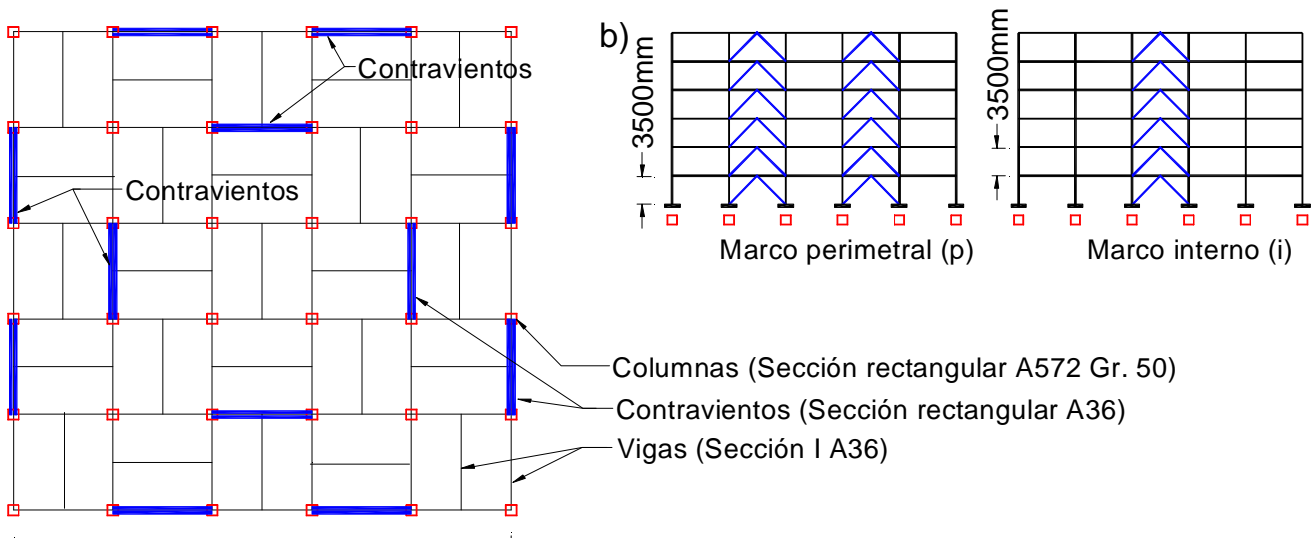

Columnas (Sección rectangular A572 Gr. 50)

Contravientos (Sección rectangular A36)

Vigas (Sección I A36)

Figura 2. Modelos estudiados: a) planta tipo, b) elevación tipo de los marcos contraventeados 
Los edificios estudiados fueron diseñados siguiendo estrictamente los criterios de las Normas Técnicas Complementarias del Reglamento de Construcciones para el Distrito Federal (NTC-RCDF-04) y fueron asociados a las mayores demandas de aceleración (zona IIIb) con el máximo factor de comportamiento sísmico $(Q=3)$ permitido para estos sistemas. En la figura 3 se presenta el espectro elástico e inelástico considerado en los análisis.

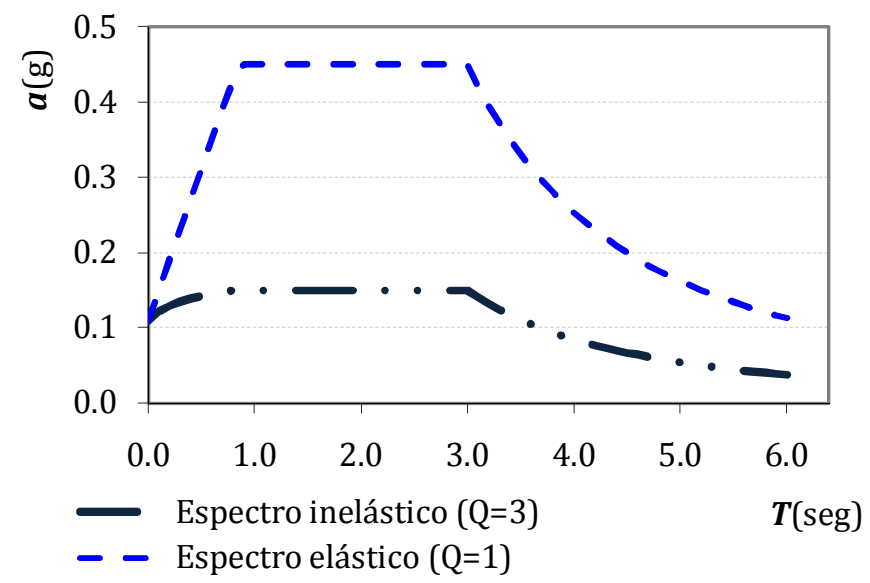

Figura 3. Espectros de diseño conforme a las NTCS-RCDF-04

Además, en su diseño se varió la contribución al cortante lateral resistente de las columnas del marco desde el 25 por ciento (que no cumple los lineamientos del RCDF-04 para diseño dúctil) al 75 por ciento. En la figura 4, se presenta el porcentaje de contribución al cortante lateral resistente de las columnas del marco de los modelos estudiados en relación a la altura total de la edificación.
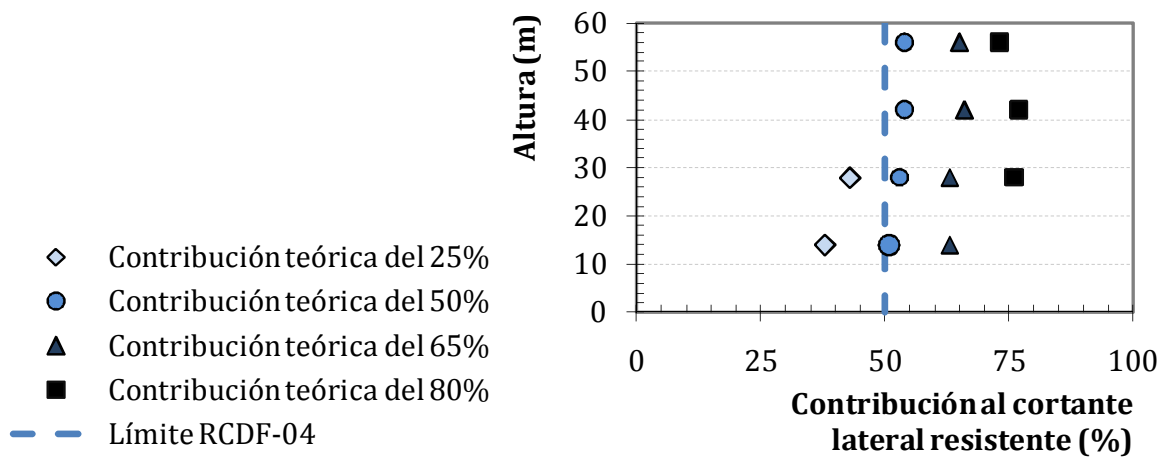

Figura 4. Identificación de los análisis realizados

En la práctica de diseño de México es común tipificar (o cambiar secciones) de columnas, vigas y contravientos un determinado número de niveles, lo que tiene ventajas constructivas evidentes, al poder estandarizar las conexiones, simplificar la supervisión en obra y agilizar el proceso constructivo. Sin embargo, para evitar cambios abruptos en la rigidez lateral de entrepiso de los marcos en elevación, el espesor de la sección cajón de las columnas se cambió cada $M$-niveles, mientras que el espesor de la sección cajón de los contravientos se cambió cada $N$-niveles. Las columnas cambian su espesor cada dos niveles en los modelos de cuatro pisos; cada tres niveles (en los niveles 1 a 6 ) y cada dos niveles (en los niveles 7 y 8 ) en los modelos de ocho pisos; y cada cuatro niveles en los modelos de doce pisos y dieciséis pisos. El sistema de contravientos no cambia de sección en los modelos de cuatro pisos; cambia cada 
cuatro niveles en los modelos de ocho pisos; cada tres niveles en los modelos de doce pisos y cada cinco niveles (entre el 1 al 10) y cada seis niveles en los modelos de dieciséis pisos. Las secciones finales se enuncian en la tabla 1 y se reportan detalladamente en Tapia (2010).

El criptograma de identificación de los modelos es Chxxpy y Chxxiy, donde Ch indica que se trata de marcos con contraventeo tipo chevrón; $x x$ indica el número de niveles; $p$ identifica que se trata de un marco perimetral con dos crujías contraventeadas y la letra $i$ si se trata de un marco interno con una sola crujía contraventeada (figura $2 \mathrm{~b}$ ) y, por último, la $y$ es el porcentaje de cortante lateral resistente contribuido por las columnas identificado por un solo dígito: un número 2 refiere al $25 \%$, 5 , el 50 por ciento; 6 el 65 por ciento; 7 el 75 por ciento; y 8 el 80 por ciento.

Tabla 1. Secciones transversales de los modelos diseñados

\begin{tabular}{|c|c|c|c|c|}
\hline \multicolumn{2}{|c|}{ Modelos } & $\begin{array}{l}\text { Columnas }(\mathrm{cm}) \\
\text { Sección cajón }\end{array}$ & $\begin{array}{c}\text { Contravientos }(\mathrm{cm}) \\
\text { Sección cajón }\end{array}$ & $\begin{array}{c}\text { Vigas } \\
\text { Sección I }\end{array}$ \\
\hline Ch4p2 y & Ancho & $20 \times 20$ & $20 \times 20$ & \\
\hline Ch4i2 & Espesor & $t_{1}=1.27 ; t_{2}=0.95$ & $t=0.64$ & W $14 \times 90.1 \mathrm{~kg} / \mathrm{m}$ \\
\hline Ch4p5 y & Ancho & $35 \times 35$ & $13 \times 13$ & \\
\hline Ch4i5 & Espesor & $t_{1}=1.59 ; t_{2}=1.27$ & $t=0.64$ & W $14 \times 101.3 \mathrm{~kg} / \mathrm{m}$ \\
\hline Ch4p7 y & Ancho & $60 \times 60$ & $13 \times 13$ & \\
\hline Ch4i7 & Espesor & $t_{1}=2.22 ; t_{2}=1.91$ & $t=0.64$ & W 16 "x $132.7 \mathrm{~kg} / \mathrm{m}$ \\
\hline Ch8p2 y & Ancho & $35 \times 35$ & $30 \times 30$ & W 16"x09 8 k $/ \mathrm{m}$ \\
\hline Ch8i2 & Espesor & $t_{1}=1.59 ; t_{2}=1.27 ; t_{3}=0.95$ & $t_{1}=3.81 ; t_{2}=3.18$ & W $10 \times 99.8 \mathrm{~kg} / \mathrm{m}$ \\
\hline Ch8p5 y & Ancho & $40 \times 40$ & $15 \times 15$ & \\
\hline Ch8i5 & Espesor & $t_{1}=1.91 ; t_{2}=1.58 ; t_{3}=1.27$ & $t_{1}=0.95 ; t_{2}=0.64$ & W $18 \times 112.9 \mathrm{~kg} / \mathrm{m}$ \\
\hline Ch8p6 y & Ancho & $65 \times 65$ & $15 \times 15$ & W 24"x1251 ko/m \\
\hline Ch8p6 & Espesor & $t_{1}=2.22 ; t_{2}=1.91 ; t_{3}=1.58$ & $t_{1}=0.95 ; t_{2}=0.64$ & W $24 \times 125.1 \mathrm{~kg} / \mathrm{m}$ \\
\hline Ch8p7 y & Ancho & $100 \times 100$ & $13 \times 13$ & \\
\hline Ch8p7 & Espesor & $t_{1}=4.12 ; t_{2}=3.49 ; t_{3}=3.18$ & $t_{l}=0.95 ; t_{2}=0.64$ & W $30 \times 13 / .4 \mathrm{~kg} / \mathrm{m}$ \\
\hline Ch12p5 y & Ancho & $\begin{array}{c}45 \times 45 \\
t=222 \cdot t=190 .\end{array}$ & $\begin{array}{c}25 \times 25 \\
t=222 \cdot t_{=}=190 .\end{array}$ & W $18 " x 1443 \mathrm{ko} / \mathrm{m}$ \\
\hline Ch12i5 & Espesor & $\begin{array}{l}t_{1}=2.22 ; t_{2}=1.90 ; \\
t_{3}=1.58 ; t_{3}=1.27\end{array}$ & $\begin{array}{c}t_{1}=2.22 ; t_{2}=1.90 \\
t_{3}=1.58\end{array}$ & W $18 \times 144.3 \mathrm{~kg} / \mathrm{m}$ \\
\hline Ch12p6 y & Ancho & $65 \times 65$ & $20 \times 20$ & \\
\hline Ch12i6 & Espesor & $\begin{array}{l}t_{1}=3.49 ; t_{2}=3.18 ; \\
t_{3}=2.86 ; t_{4}=2.54\end{array}$ & $\begin{array}{c}t_{1}=1.27 ; t_{2}=0.95 \\
t_{3}=0.64\end{array}$ & W 24”x217.8 kg/m \\
\hline & Ancho & $100 \times 100$ & $12 \times 12$ & \\
\hline Ch12p8 & Espesor & $\begin{array}{l}t_{1}=4.45 ; t_{2}=4.13 ; \\
t_{3}=3.82 ; t_{4}=3.49\end{array}$ & $\begin{array}{c}t_{1}=1.27 ; t_{2}=0.95 ; \\
t_{3}=0.64\end{array}$ & W $27 ” \times 240.1 \mathrm{~kg} / \mathrm{m}$ \\
\hline Ch16p5 y & Ancho & $50 \times 50$ & $35 \times 35$ & \\
\hline Ch16i5 & Espesor & $\begin{array}{l}t_{1}=2.54 ; t_{2}=1.91 ; \\
t_{3}=1.58 ; t_{4}=1.27\end{array}$ & $\begin{array}{c}t_{1}=3.49 ; t_{2}=3.18 \\
t_{3}=2.86\end{array}$ & W $21 " x 150.9 \mathrm{~kg} / \mathrm{m}$ \\
\hline Ch16p6 y & Ancho & $60 \times 60$ & $20 \times 20$ & \\
\hline Ch16i6 & Espesor & $\begin{array}{l}t_{1}=2.86 ; t_{2}=2.54 ; \\
t_{3}=2.22 ; t_{4}=1.91\end{array}$ & $\begin{array}{c}t_{1}=1.58 ; t_{2}=1.27 ; \\
t_{3}=0.95\end{array}$ & W 24”x217.8 kg/m \\
\hline & Ancho & $120 \times 120$ & $15 \times 15$ & \\
\hline $\begin{array}{l}\text { ChIop8 y } \\
\text { Ch16i8 }\end{array}$ & Espesor & $\begin{array}{l}t_{1}=4.45 ; t_{2}=4.13 ; \\
t_{3}=3.81 ; t_{4}=3.49\end{array}$ & $\begin{array}{c}t_{1}=1.27 ; t_{2}=0.95 \\
t_{3}=0.64\end{array}$ & W 24”x217.8 kg/m \\
\hline
\end{tabular}

\section{ANÁLISIS ESTÁTICOS NO LINEALES}

Se realizaron análisis estáticos no lineales ante carga monótona creciente con el programa Drain2DX (Prakash et al. 1992), considerando una distribución triangular de carga lateral consistente con el método estático que establecen las Normas Técnicas del RCDF-04. 
En las siguientes figuras se presentan las instantáneas de fluencia distinguiendo por color la escala de magnitud de deformación inelástica en los elementos normalizada con la magnitud máxima de fluencia en trabes y columnas o el máximo acortamiento o extensión en contravientos. En las crujías contraventeadas, las diagonales del lado izquierdo están en tensión con extensiones axiales, mientras que las diagonales del lado derecho están en compresión con acortamientos asociados al pandeo. En cada figura se incluye la distorsión global en porcentaje obtenida como la deformación en lo alto del modelo y la base dividida entre la altura total.

La magnitud final de fluencia considerada en este estudio toma en cuenta la capacidad teórica real que puede desarrollar cada elemento, es decir, no es la magnitud reportada en el último paso del archivo de salida. Los límites teóricos para el pandeo de los contravientos se definieron considerando las ecuaciones derivadas por Kemp (1996) de una investigación experimental, conforme al procedimiento que se detalla en Tapia (2005).

\section{Modelos con aportación de columnas al cortante lateral resistente alrededor del $25 \%$}

Los modelos $C h 4 p 2$, Ch4i2, Ch8p2 y $C h 8 i 2$ fueron diseñados con columnas que proveen una aportación al cortante lateral resistente mayor al $25 \%$, pero menor al $50 \%$ usando perfiles laminados de acero comerciales. Note que esta contribución no satisface la aportación mínima solicitada por el RCDF04 para comportamiento dúctil; sin embargo, su inclusión en el estudio permite valorar la conveniencia del requisito propuesto (del 50\%), definido con base en la experiencia y sentido común más que por estudios específicos sobre el balance de rigidez en estos sistemas estructurales que puede guiar al mecanismo de colapso supuesto y la capacidad de deformación asociada a él.

Estos modelos (figura 5) iniciaron su comportamiento no lineal con rotaciones en las vigas. Sin embargo, el mecanismo de piso débil predominó posteriormente con fluencia de columnas en ambos extremos en los entrepisos inferiores. En este sentido, estos resultados sugieren que la recomendación existente en el RCDF-04, sobre no permitir el diseño dúctil de marcos contraventeados con una contribución baja al cortante lateral resistente de las columnas, es adecuada.
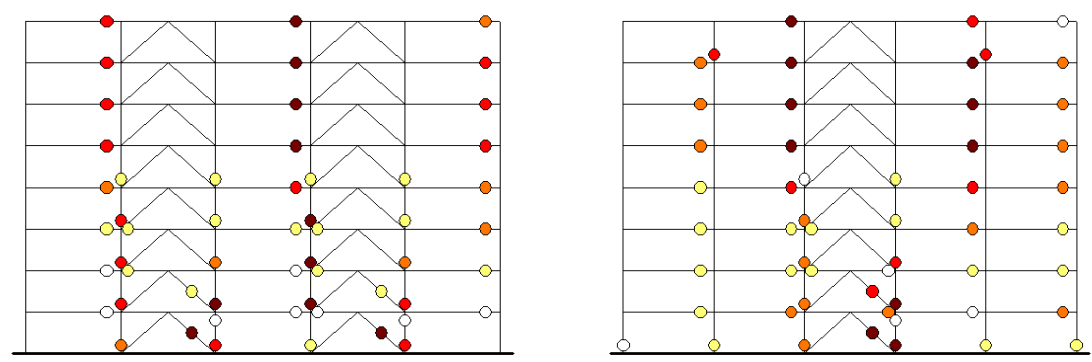

$0.00-0.20 \bigcirc$

$0.20-0.40 \bigcirc$

$0.40-0.60 \bigcirc$

$0.60-0.80$

$0.80-1.00$

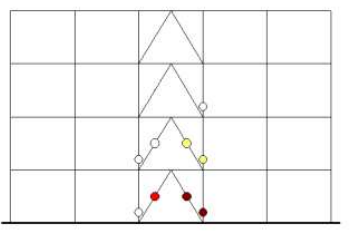

Figura 5. Mecanismos de falla representativos de los marcos cuyas columnas resisten alrededor del $25 \%$ de la carga sísmica total

\section{Modelos con aportación de columnas al cortante lateral resistente alrededor del $50 \%$}

La fluencia inicial de los modelos de cuatro, ocho, doce y dieciséis niveles diseñados para que sus columnas contribuyan alrededor del $50 \%$ se presentan en la figura 6 . Estos modelos cumplen todos los requisitos estipulados en el reglamento para comportamiento dúctil. 
Los modelos de baja altura (Ch4 y Ch8) iniciaron su comportamiento inelástico con el pandeo de los contravientos en compresión (figura 6a). En contraste, los modelos altos iniciaron su fluencia en las vigas (figura $7 \mathrm{a}$ ).

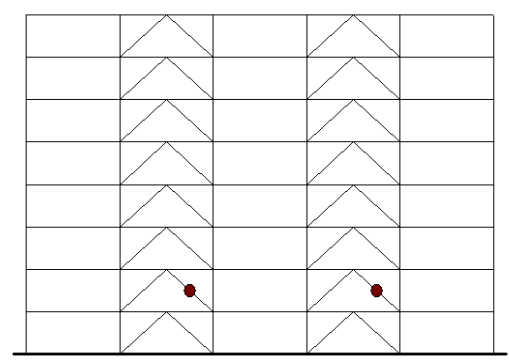

a) $\delta_{\text {global }}=0.231(\%)$

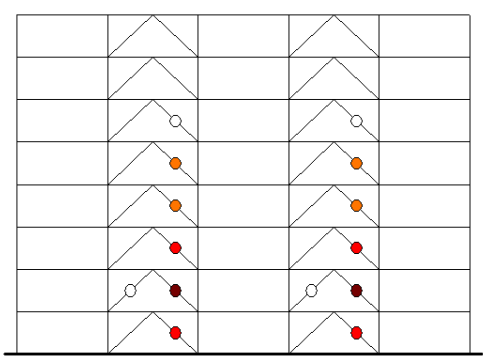

b) $\delta_{\text {global }}=0.349(\%)$

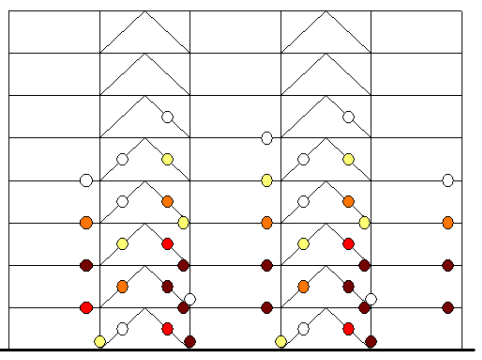

c) $\delta_{\text {global }}=0.648(\%)$

Figura 6. Formación del mecanismo de colapso del modelo Ch8p5

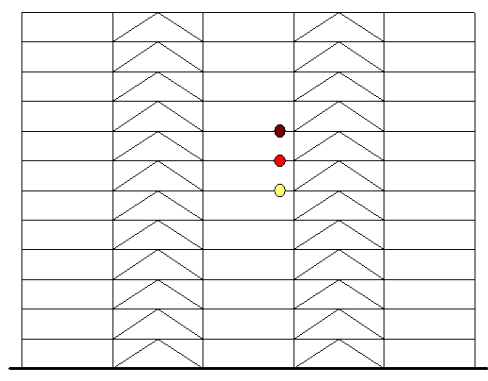

a) $\delta_{\text {global }}=0.305(\%)$

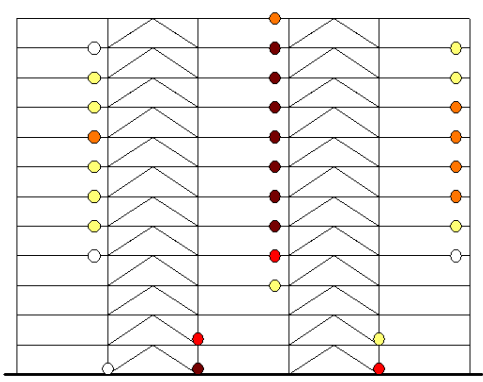

b) $\delta_{\text {global }}=0.545(\%)$

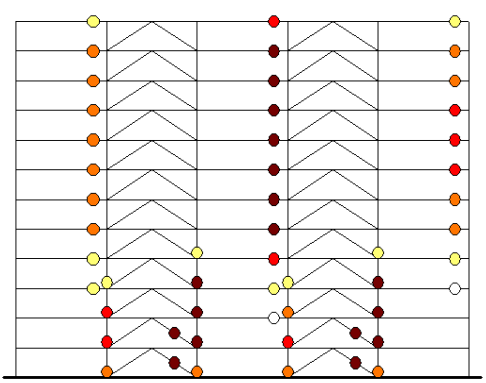

c) $\delta_{\text {global }}=0.883(\%)$

Figura 7. Formación del mecanismo de colapso del modelo Ch12p5

Los mecanismos de colapso de los modelos de baja altura ( $C h 4$ y $C h 8$ ) coinciden razonablemente con el mecanismo supuesto en las premisas de diseño (figura 6c), iniciando con la plastificación de los contravientos por el pandeo en compresión o fluencia por tensión, rotaciones plásticas en vigas y finalmente rotaciones plásticas en las columnas de la base.

Sin embargo, los mecanismos de colapso obtenidos se modifican con el aumento de la altura. A pesar de las rotaciones plásticas en las vigas, pocos contravientos se pandean por compresión y se desarrollan fluencias en los extremos de algunas columnas, especialmente en el modelo Ch16 (figura 7c). Estos resultados se aproximan a un mecanismo de colapso de piso débil sin que exista una distribución uniforme de las fluencias en la altura, lo que es completamente diferente a las suposiciones realizadas durante el proceso de diseño.

\section{Modelos con aportación de columnas al cortante lateral resistente alrededor del $65 \%$}

Para este balance de resistencia, el pandeo de los contravientos supuesto se extiende hasta los modelos de doce niveles, que coinciden con las hipótesis de diseño hasta la formación final del mecanismo (figura 8). Los modelos de baja altura exhiben una distribución de fluencias más uniforme en la altura que los modelos altos (no incluidos, pero pueden ser consultados en Tapia y Tena 2008, Tapia 2011).

Por otra parte, los modelos de dieciséis niveles no desarrollan en su totalidad el mecanismo de colapso supuesto en el RCDF-04 (figura 9). El comportamiento inelástico se presenta en las vigas 
predominantemente sin pandeo del contraviento en compresión en los entrepisos superiores y articulaciones en columnas en los primeros dos niveles.

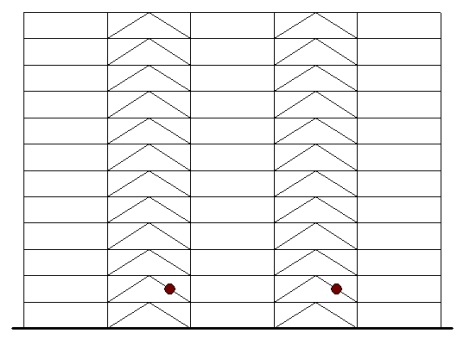

a) $\delta_{\text {global }}=0.273(\%)$

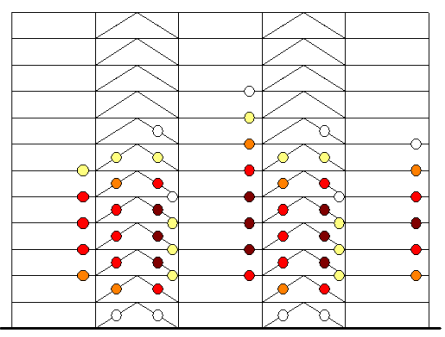

b) $\delta_{\text {global }}=0.513(\%)$

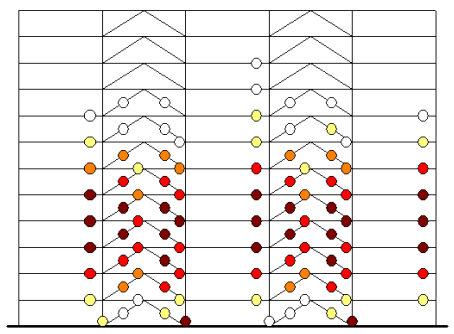

c) $\delta_{\text {global }}=0.996(\%)$

Figura 8. Formación del mecanismo de colapso del modelo Ch12p6

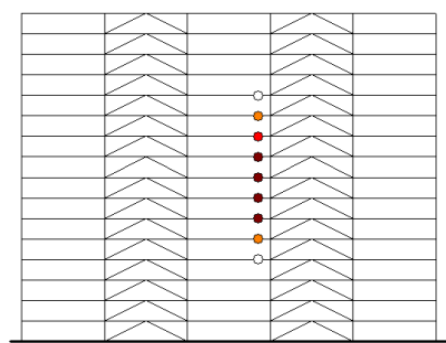

a) $\delta_{\text {global }}=0.331(\%)$

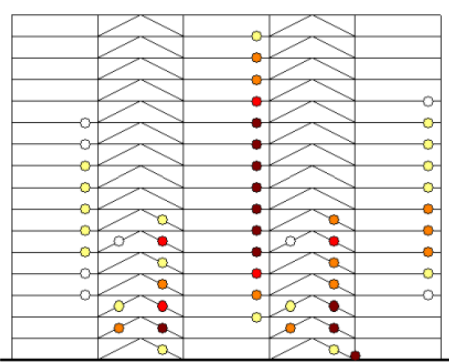

b) $\delta_{\text {global }}=0.481(\%)$

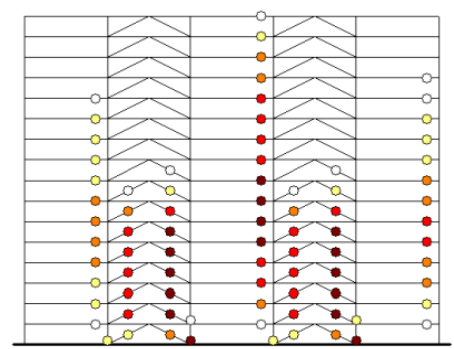

c) $\delta_{\text {global }}=0.729(\%)$

Figura 9. Formación del mecanismo de colapso del modelo Ch16p6

\section{Modelos con aportación de columnas al cortante lateral resistente alrededor del 75\%}

La formación del mecanismo de fluencia del modelo de 16 niveles que fue diseñado para que las columnas resistan alrededor del $75 \%$ del cortante lateral resistente se ilustra en la figura 10 . Se observa que la respuesta inelástica inicia con la plastificación de los contravientos por pandeo en compresión o la fluencia por tensión y subsecuentemente la formación de rotaciones plásticas en vigas. Todos los modelos exhiben una distribución uniforme en la altura de las fluencias asociada al mecanismo de colapso columna fuerte - viga débil - contraviento más débil, supuesto durante el proceso de diseño (Tapia y Tena 2008, Tapia 2011).

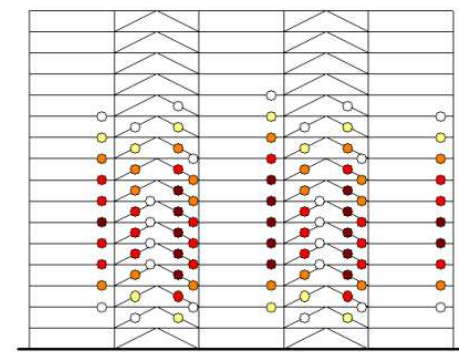

a) $\delta_{\text {global }}=0.457(\%)$

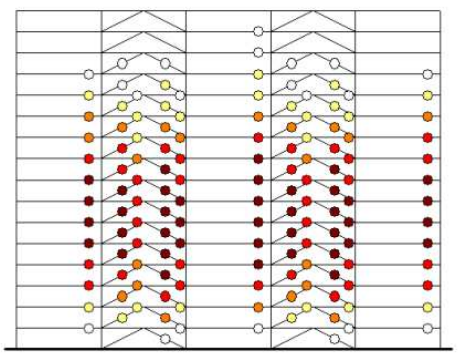

b) $\delta_{\text {global }}=0.995(\%)$

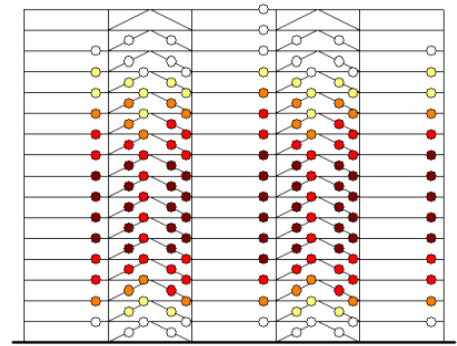

c) $\delta_{\text {global }}=1.569(\%)$

Figura 10. Formación del mecanismo de colapso del modelo Ch16p8 
Las columnas permanecen elásticas con excepción de algunas fluencias en el modelo Ch12. El comportamiento inelástico de las columnas se relaciona con la condición de columnas empotradas y con el incremento de rigidez requerido para estos marcos.

Las rotaciones máximas en vigas y columnas, así como las deformaciones axiales en contravientos se incluyen en la tabla 2. Las magnitudes presentadas toman en cuenta la reducción en los pasos del análisis no lineal producto de considerar las rotaciones y acortamiento teóricas reales de las secciones estructurales siguiendo los criterios presentados en Kemp (1996).

Tabla 2. Rotaciones, alargamientos y acortamientos máximos de los modelos

\begin{tabular}{lcccc}
\hline Modelo & $\begin{array}{c}\text { Rotación en } \\
\text { columnas } \\
\text { (rad.) }\end{array}$ & $\begin{array}{c}\text { Rotación en } \\
\text { trabes (rad.) }\end{array}$ & $\begin{array}{c}\text { Alargamiento } \\
\text { (cm.) }\end{array}$ & $\begin{array}{c}\text { Acortamiento } \\
\text { (cm.) }\end{array}$ \\
\hline Ch4p2 & 0.005 & 0.000 & 0.00 & 1.91 \\
Ch4i2 & 0.005 & 0.000 & 0.00 & 1.80 \\
Ch4p5 & 0.004 & 0.009 & 2.23 & 3.35 \\
Ch4i5 & 0.009 & 0.014 & 2.80 & 4.20 \\
Ch4p6 & 0.008 & 0.016 & 3.67 & 4.87 \\
Ch4i6 & 0.002 & 0.013 & 2.62 & 3.84 \\
Ch8p2 & 0.020 & 0.036 & 0.00 & 4.47 \\
Ch8i2 & 0.023 & 0.025 & 0.00 & 5.52 \\
Ch8p5 & 0.012 & 0.014 & 2.30 & 4.74 \\
Ch8i5 & 0.004 & 0.009 & 1.44 & 3.37 \\
Ch8p6 & 0.000 & 0.022 & 6.95 & 7.81 \\
Ch8i6 & 0.000 & 0.016 & 5.28 & 6.08 \\
Ch8p7 & 0.000 & 0.014 & 2.26 & 3.60 \\
Ch8i7 & 0.000 & 0.022 & 3.95 & 5.53 \\
Ch12p5 & 0.005 & 0.016 & 0.00 & 0.10 \\
Ch12i5 & 0.011 & 0.009 & 0.48 & 1.54 \\
Ch12p6 & 0.017 & 0.031 & 7.12 & 7.46 \\
Ch12i6 & 0.016 & 0.032 & 7.51 & 7.85 \\
Ch12p8 & 0.009 & 0.029 & 6.72 & 7.09 \\
Ch12i8 & 0.001 & 0.021 & 4.95 & 5.33 \\
Ch16p5 & 0.038 & 0.086 & 0.24 & 0.98 \\
Ch16i5 & 0.033 & 0.056 & 0.24 & 0.82 \\
Ch16p6 & 0.002 & 0.008 & 0.66 & 0.87 \\
Ch16i6 & 0.002 & 0.007 & 1.10 & 1.60 \\
Ch16p8 & 0.000 & 0.024 & 5.03 & 5.38 \\
Ch16i8 & 0.000 & 0.026 & 5.47 & 5.81 \\
\hline & & & & \\
\hline
\end{tabular}

\section{CORTANTE MÍNIMO RESISTIDO POR EL MARCO}

En la literatura se han presentado estudios en modelos de poca altura (por ejemplo, Bruneau et al. 1998) en los que el mecanismo de colapso coincide aceptablemente bien cuando la contribución al cortante lateral resistente de las columnas del marco es cercana al 50\% suponiendo que, a partir de un patrón de cargas iniciales, se obtendrán mecanismos predecibles (columna fuerte - viga débil contraviento más débil), como ocurre en el modelo Ch4. Sin embargo, diversos estudios (Khatib et al. 1998, Remennikov y Walpole 1998, Elghazouli 2003, MacRae et al. 2004, Tapia 2005, Tapia y Tena 2009, Fell et al. 2010) han demostrado que se pueden desarrollar mecanismos de piso débil en edificios de mediana altura. 
Los resultados presentados en este estudio sugieren que existe una relación entre la altura de la estructura y la relación entre la contribución al cortante lateral resistente de las columnas del marco y el sistema de contravientos con el mecanismo de colapso desarrollado, que no está considerada en los reglamentos vigentes.

Con este fundamento, considerando los resultados obtenidos, se propone una estrategia de diseño para definir una contribución lateral al cortante resistente mínimo que deben aportar las columnas del marco en relación al sistema de contravientos, a fin de obtener un mecanismo de colapso columna fuerte viga débil - contraviento más débil. La propuesta está en función de la relación de esbeltez del edificio y de los esfuerzos de fluencia de los elementos, conforme se establece en la ecuación 1:

$$
\frac{V_{R C o l}}{V_{\text {Tot }}}=0.50+0.05 \frac{H}{B} \sqrt{\frac{F_{y C o l}}{F_{y \text { Diag }}}}
$$

donde $H$ es la altura del edificio, $B$ es la mínima dimensión en planta en la dirección de interés, $F_{y}$ Diag el esfuerzo de fluencia de los contravientos y $F_{y} \mathrm{Col}$ el esfuerzo de fluencia de las columnas. La ecuación propuesta fue determinada considerando las demandas símicas en suelos blandos, por lo que conclusiones diferentes se podrían obtener en suelos con una sismicidad de sitio diferente.

En la figura 11 se ejemplifica el comportamiento de las ecuaciones sugeridas, coincidente a las observaciones de este estudio, donde las columnas se modelaron con acero tipo A-572 Gr. 50, mientras que los contravientos son de acero A36. Note que en el razonamiento propuesto, al considerar que ambos tipos de elementos fueran de acero A36, el límite de aportación del cortante resistente de las columnas sufriría una ligera reducción por considerar que las columnas podrían articularse antes que los resultados obtenidos en este estudio. Asimismo, se hace notar que cuando se considera acero de alta resistencia en contravientos, se debe prestar mayor atención a la posibilidad de que se presente pandeo local y pandeo lateral en el caso de las trabes.
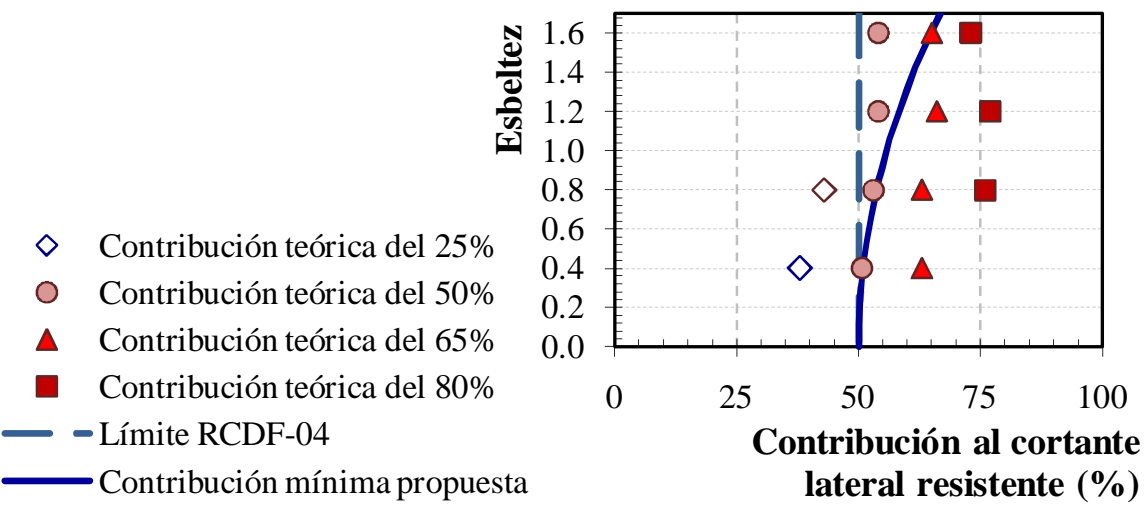

Figura 11.Contribución mínima al cortante lateral resistente de las columnas

Así, esta ecuación permite estimar crudamente el porcentaje mínimo de la carga sísmica que las columnas deben tomar para obtener poco o nulo daño y concentrar las deformaciones inelásticas en el sistema de contravientos primeramente y en las trabes. 


\section{FACTOR DE REDUCCIÓN POR DUCTILIDAD}

A partir de las curvas cortante - distorsión obtenidas de los análisis no lineales ante carga monótona creciente, es posible definir una curva elasto-plástica idealizada del comportamiento del sistema, prolongando una línea con la pendiente del intervalo elástico hasta otra línea horizontal que señala la máxima magnitud al cortante alcanzada. En la figura 12 se ejemplifican las curvas obtenidas para el modelo Ch8p50.
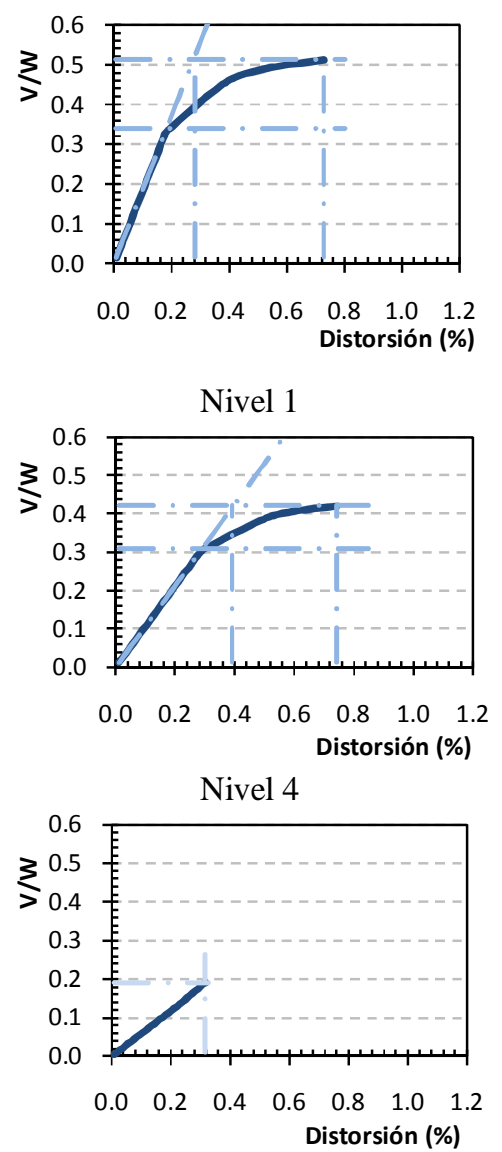

Nivel 7

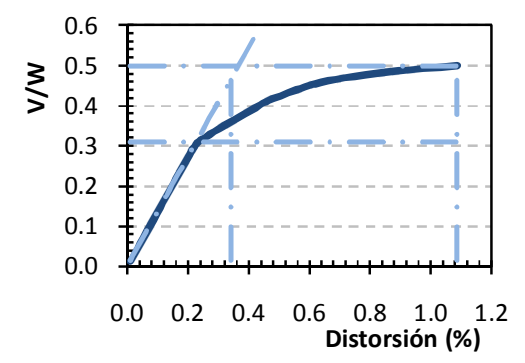

Nivel 2

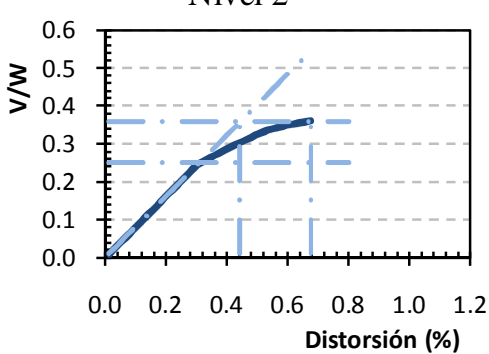

Nivel 5

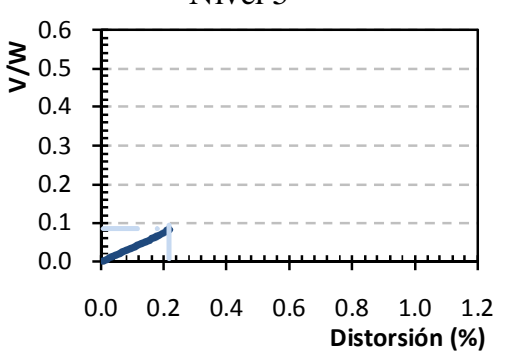

Nivel 8

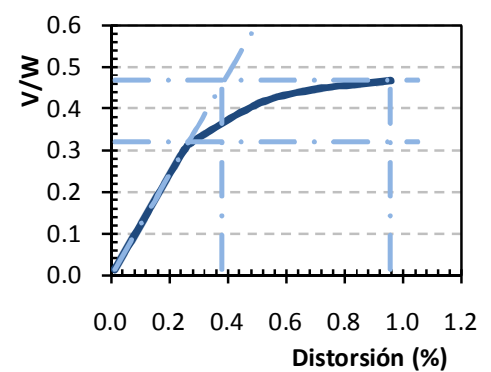

Nivel 3

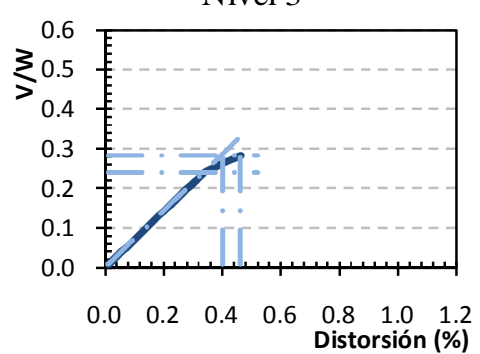

Nivel 6

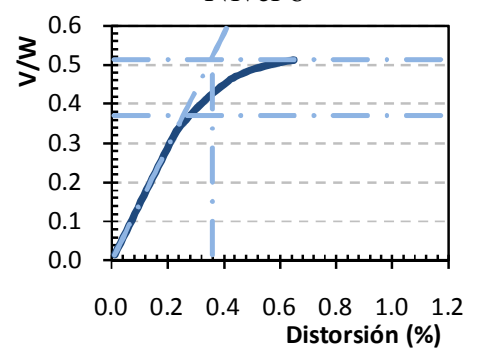

Global

Figura 12. Curvas cortante - distorsión del modelo Ch8p50

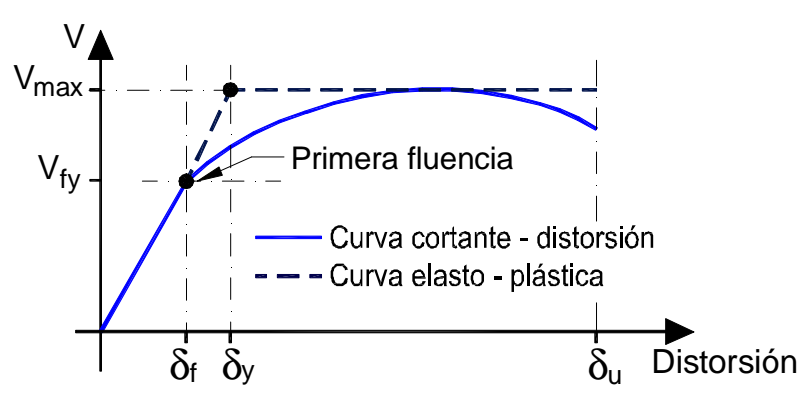

Figura 13. Definición de la distorsión en primera fluencia y la distorsión de fluencia 
La distorsión teórica definida de las curvas bilineales, que es considerada usualmente en los reglamentos para la definición de la distorsión de fluencia, está definida como $\delta_{y}$. Se obtuvieron las distorsiones de todos los entrepisos $\delta_{i}$ para compararlas con la distorsión global $\delta_{g}$. La distorsión global se calculó con el cortante basal y la distorsión entre la base y la azotea del edificio. Así, es posible obtener dos diferentes magnitudes de ductilidad: una relacionada con las distorsiones a la primera fluencia $\delta_{f}$ obtenida de las curvas cortante - distorsión y una ductilidad teórica obtenida con la distorsión de fluencia $\delta_{y}$ de la curva elasto - plástica perfecta (figura 13).

\section{Distorsiones}

En la tabla 3 se reportan las distorsiones de fluencia obtenidas de considerar la curva global, $\delta_{y g}$, el promedio de los entrepisos, $\delta_{y e}$, y el promedio de ambas, $\delta_{y p}$. Estos resultados no incluyen al primer nivel por la condición de frontera impuesta, ni las distorsiones de los entrepisos que evidenciaron un comportamiento elástico.

Tabla 3. Distorsiones promedio (\%) de los modelos

\begin{tabular}{|c|c|c|c|c|c|c|c|c|c|}
\hline \multirow{2}{*}{ Modelo } & \multicolumn{3}{|c|}{$\begin{array}{l}\text { Distorsión de fluencia } \delta_{f} \text { de la } \\
\text { curva cortante-distorsión }\end{array}$} & \multicolumn{3}{|c|}{$\begin{array}{l}\text { Distorsión de fluencia } \delta \text {, de la } \\
\text { curva primaria idealizada }\end{array}$} & \multicolumn{3}{|c|}{ Distorsión última $\delta_{u}$} \\
\hline & $\begin{array}{c}\text { Global } \\
\delta_{f g}\end{array}$ & $\begin{array}{c}\text { Entrepiso } \\
\delta_{f e}\end{array}$ & $\begin{array}{c}\text { Promedio } \\
\delta_{f p}\end{array}$ & $\begin{array}{c}\text { Global } \\
\delta_{y g}\end{array}$ & $\begin{array}{c}\text { Entrepiso } \\
\delta_{y e}\end{array}$ & $\begin{array}{c}\text { Promedio } \\
\delta_{y p}\end{array}$ & $\begin{array}{c}\text { Global } \\
\delta_{u g}\end{array}$ & $\begin{array}{c}\text { Entrepiso } \\
\delta_{u e}\end{array}$ & $\begin{array}{c}\text { Promedio } \\
\delta_{u p}\end{array}$ \\
\hline Ch4p5 & 0.20 & 0.22 & 0.21 & 0.30 & 0.35 & 0.33 & 0.86 & 0.99 & 0.93 \\
\hline Ch4p6 & 0.29 & 0.29 & 0.29 & 0.40 & 0.40 & 0.40 & 1.27 & 1.30 & 1.29 \\
\hline Ch8p5 & 0.25 & 0.28 & 0.26 & 0.33 & 0.39 & 0.36 & 0.65 & 0.78 & 0.72 \\
\hline Ch8p6 & 0.27 & 0.31 & 0.29 & 0.37 & 0.41 & 0.39 & 1.16 & 1.40 & 1.28 \\
\hline Ch8p7 & 0.25 & 0.26 & 0.26 & 0.39 & 0.41 & 0.40 & 1.49 & 1.61 & 1.55 \\
\hline Ch12p5 & 0.43 & 0.46 & 0.44 & 0.59 & 0.61 & 0.60 & 0.95 & 1.00 & 0.98 \\
\hline Ch12p6 & 0.34 & 0.37 & 0.36 & 0.44 & 0.47 & 0.45 & 1.00 & 1.21 & 1.11 \\
\hline Ch12p8 & 0.24 & 0.25 & 0.25 & 0.38 & 0.40 & 0.39 & 1.22 & 1.29 & 1.25 \\
\hline Ch16p5 & 0.42 & 0.45 & 0.43 & 0.62 & 0.66 & 0.64 & 1.54 & 1.62 & 1.58 \\
\hline Ch16p6 & 0.36 & 0.40 & 0.38 & 0.46 & 0.49 & 0.47 & 0.63 & 0.67 & 0.65 \\
\hline Ch16p8 & 0.29 & 0.29 & 0.29 & 0.42 & 0.44 & 0.43 & 1.32 & 1.38 & 1.35 \\
\hline
\end{tabular}

Las NTCS-RCDF-04 estipulan para la revisión por limitación de daños ante el sismo de servicio que la distorsión lateral de entrepiso sea menor a $\delta_{y-p e r m}=0.004$ cuando no hay elementos no estructurales incapaces de soportar deformaciones apreciables y, en lo referente a prevención contra colapso, las diferencias entre los desplazamientos laterales de pisos consecutivos no debe exceder $\delta_{u \text {-perm }}=0.015 h$ en marcos de acero con contravientos concéntricos, donde $h$ es la altura del entrepiso en cuestión.

En la figura 14a se presentan las distorsiones de fluencia promedio $\delta_{f p}(\%)$ obtenidas de las curvas cortante-distorsión y son comparadas con respecto a $\delta_{y-p e r m}$. En la figura $14 \mathrm{~b}$ se presentan los resultados de las distorsiones promedio $\delta_{y p}(\%)$ obtenidas a partir de las curvas elasto-plásticas idealizadas. Las distorsiones últimas obtenidas promedio (\%) se presentan en la figura $14 \mathrm{c}$ y se comparan con la distorsión permisible establecida en el Apéndice A para seguridad contra colapso (NTCS-RCDF-04).

Las distorsiones de fluencia $\delta_{y p}$, obtenidas a partir de las curvas idealizadas de los modelos resultan cercanas a la distorsión permisible, por lo que la propuesta reglamentaria es razonable para fines de diseño. En cambio, el análisis de las distorsiones últimas promedio obtenidas, $\delta_{u p}$, (tabla 1 , figura $6 \mathrm{c}$ ) sugieren una revisión de la distorsión límite establecida en las NTCS-RCDF-04 para la revisión del estado de prevención de colapso, dado que en la mayoría de los modelos ésta resultó menor a $\delta_{u-p e r m}=0.015$. No 
se observa una dependencia o relación directa entre $\delta_{u}$ y: a) el porcentaje de contribución al cortante sísmico que resisten las columnas ó, b) la altura total y/o esbeltez de los edificios.

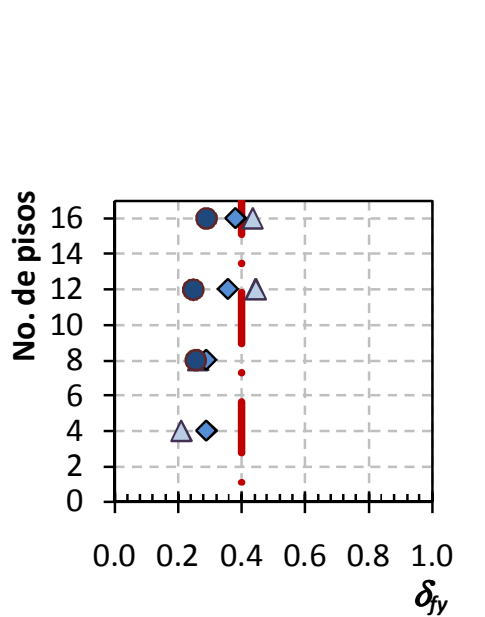

a) Distorsión de fluencia de la curva cortante - distorsión (\%)

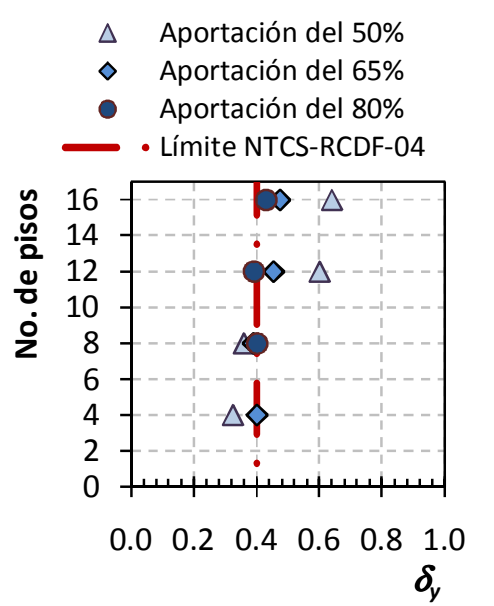

b) Distorsión de fluencia teórica de la curva primaria idealizada $(\%)$

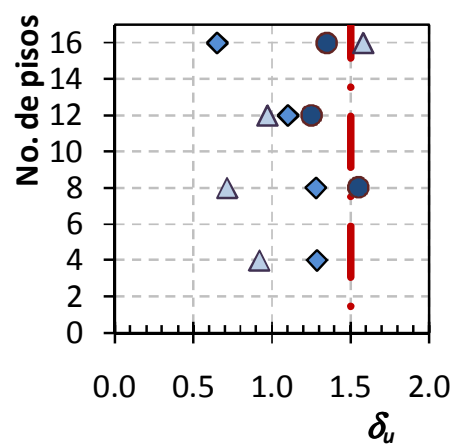

c) Distorsión última $(\%)$

Figura 14. Distorsiones de diseño (\%) y su relación con el número de pisos de los modelos

\section{Reducción por comportamiento no lineal}

A partir de las curvas carga-distorsión obtenidas de los análisis pushover y de la elasto-plástica idealizada se obtuvieron las ductilidades (demandas de deformación) que pueden desarrollar los modelos, considerando la distorsión de fluencia $\delta_{f}$ de la curva cortante - distorsión, la distorsión teórica $\delta_{y}$ de la curva primaria idealizada y la distorsión última $\delta_{u}$; es decir, el cociente de la demanda de resistencia elástica entre la demanda de resistencia inelástica (ecuación 2).

$$
\mu=\frac{F_{y}\left(\mu_{i}=1\right)}{F_{y}\left(\mu_{i}=\mu\right)}
$$

En la tabla 4 se reportan la ductilidad global $\mu_{g}$ y la ductilidad promedio de los entrepisos $\mu_{e}$, que no considera la ductilidad del entrepiso de planta baja (por su condición de frontera impuesta) ni de los niveles de comportamiento lineal $(\mu \leq 1)$.

Todos los modelos que fueron diseñados para que las columnas del marco aporten al menos el 50\% del cortante lateral resistente conforme a las Normas Técnicas (50\%) están asociados a fallas frágiles con ductilidad nula, prácticamente, $\mu \approx 1.0$ (figura 15 ). Debido a que la distorsión de fluencia $\delta_{y}$ obtenida de la curva primara idealizada es en todos los casos mayor a la asociada a la primera fluencia $\delta_{f}$ de la curva cortante - distorsión, no es extraño que las ductilidades asociadas a esta última sean sensiblemente mayores hasta $\mu=6.0$. En ambos casos, además de denotar una dependencia al porcentaje de contribución al cortante lateral resistente, los resultados son función de la altura del modelo, de manera que entre mayor sea la altura, menor será la ductilidad desarrollada.

Las demandas de deformación asociadas a los modelos de mediana y baja altura que cumplen los requisitos del porcentaje de aportación mínimo al cortante lateral antes discutido, coinciden 
aceptablemente con la ductilidad de diseño $Q=3$ (RCDF-04). Sin embargo, esta tendencia difiere con el aumento de los niveles del modelo. Los resultados apuntan al hecho que la reducción de fuerza por este concepto estipulada en los códigos debería ser entonces dependiente de la altura de los modelos.

Tabla 4. Ductilidades desarrolladas por los modelos

\begin{tabular}{|c|c|c|c|c|c|c|c|}
\hline \multirow{2}{*}{ Modelo } & \multirow{2}{*}{$\begin{array}{c}\text { Esbeltez } \\
\text { del modelo } \\
H / B\end{array}$} & \multicolumn{3}{|c|}{$\begin{array}{l}\text { Ductilidad obtenida de las } \\
\text { curvas cortante - distorsión }\end{array}$} & \multicolumn{3}{|c|}{$\begin{array}{l}\text { Ductilidad teórica de las } \\
\text { curvas primaria idealizada }\end{array}$} \\
\hline & & $\begin{array}{c}\text { Global } \\
\mu_{g}\end{array}$ & $\begin{array}{c}\text { Entrepiso } \\
\mu_{e}\end{array}$ & $\begin{array}{c}\text { Promedio } \\
\mu_{p}\end{array}$ & $\begin{array}{c}\text { Global } \\
\mu_{g}\end{array}$ & $\begin{array}{c}\text { Entrepiso } \\
\mu_{e}\end{array}$ & $\begin{array}{c}\text { Promedio } \\
\mu_{p}\end{array}$ \\
\hline Ch4p2 & \multirow{3}{*}{0.40} & 1.17 & 1.00 & 1.08 & 1.08 & 1.00 & 1.04 \\
\hline Ch4p5 & & 4.20 & 3.19 & 3.69 & 2.87 & 2.17 & 2.52 \\
\hline Ch4p6 & & 4.35 & 4.45 & 4.40 & 3.18 & 3.22 & 3.20 \\
\hline Ch8p2 & \multirow{4}{*}{0.80} & 1.05 & 1.00 & 1.03 & 1.00 & 1.00 & 1.00 \\
\hline Ch8p5 & & 2.64 & 2.93 & 2.79 & 1.97 & 2.06 & 2.32 \\
\hline Ch8p6 & & 4.34 & 4.57 & 4.46 & 3.14 & 2.72 & 2.93 \\
\hline Ch8p7 & & 5.96 & 5.93 & 5.95 & 3.82 & 4.09 & 3.95 \\
\hline Ch12p5 & \multirow{3}{*}{1.20} & 2.00 & 1.94 & 1.97 & 1.36 & 1.38 & 1.37 \\
\hline Ch12p6 & & 2.95 & 3.36 & 3.15 & 2.27 & 2.31 & 2.29 \\
\hline Ch12p8 & & 5.00 & 4.79 & 4.90 & 3.21 & 3.12 & 3.16 \\
\hline Ch16p5 & \multirow{3}{*}{1.60} & 1.99 & 1.90 & 1.95 & 1.86 & 1.77 & 1.81 \\
\hline Ch16p6 & & 1.76 & 1.63 & 1.70 & 1.37 & 1.36 & 1.37 \\
\hline Ch16p8 & & 4.59 & 4.51 & 4.55 & 3.14 & 2.97 & 3.06 \\
\hline
\end{tabular}

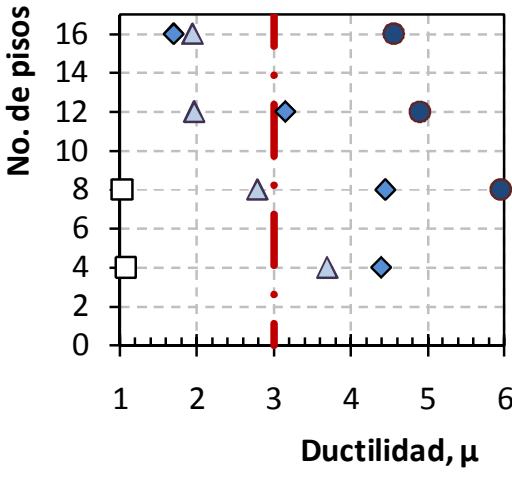

a) Ductilidad de la curva cortante distorsión

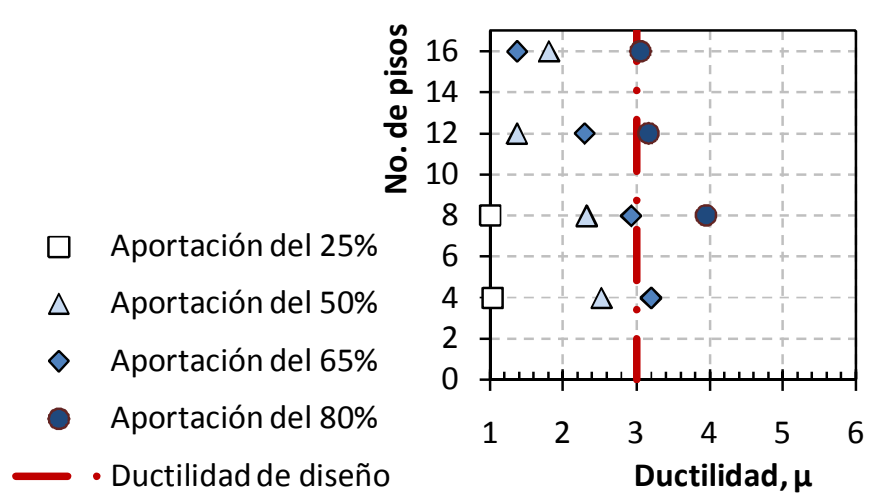

b) Ductilidad de la curva primaria idealizada

Figura 15. Comportamiento de la ductilidad de los modelos en relación a la altura

\section{Tendencia de reglamentos vigentes}

En los reglamentos de diseño, las reducciones en fuerzas producidas por la disipación de energía histerética se obtienen al permitir un comportamiento no lineal en la estructura y se toman en cuenta a partir de factores de reducción. Algunos reglamentos internacionales definen la magnitud de la ductilidad de diseño en función de la altura y en función del periodo fundamental del terreno, lo que guarda congruencia con los resultados obtenidos y reconociendo que los marcos con contravientos tradicionales 
son más propensos a la formación de pisos débiles con el aumento de la altura (Miranda 1994, MacRae 2004, Izvernari 2007, Tapia y Tena 2008).

El cuerpo principal del Reglamento Nacional de Edificios de Canadá (CNBC-05) presenta magnitudes constantes de los factores de reducción de fuerzas sísmicas con fines de diseño por concepto de ductilidad $\left(R_{d}\right)$. Estos factores reducen directamente el espectro de diseño, de manera que son equivalentes al factor de comportamiento sísmico reducido $Q$ ' del RCDF-04. Sin embargo, el suplemento de las CSA-06 (CAN/CSA-06) presenta una propuesta $\mu=3.0$ para marcos dúctiles de acero con contraviento concéntrico (MD) con una reducción del 3\% por metro después de los $32 \mathrm{~m}$ de altura del modelo. Cuando se trata de marcos contraventeados de ductilidad limitada (LD), la reducción es $\mu=2.0$ con una modificación a los $48 \mathrm{~m}$ con una pendiente del $2 \%$ por metro.

Los reglamentos en estudio coinciden en establecer para marcos dúctiles con contraventeo concéntrico una reducción de fuerzas no mayor a 3.0. Sin embargo, algunos reconocen que se podrían alcanzar ductilidades mayores para marcos con detallado dúctil. El RCDF-04 sugiere una ductilidad igual a 4.0, mientras que las provisiones para el diseño sísmico del 2001 del Euro-código 8 y el reglamento canadiense reconocen la posibilidad de que marcos dúctiles momento resistentes alcancen ductilidades de hasta 5.0 para ofrecer un intervalo realista del comportamiento de las estructuras (Mitchell et al. 2003, Tapia y Tena 2010).

Con base en los resultados discutidos, se ha propuesto una ecuación simplificada para la determinación del factor de reducción en marcos dúctiles de acero con contraventeo concéntrico (ecuación 3 ), en función de la relación de esbeltez $H / B$ de la estructura, que supone una disminución lineal entre 0.80 $<H / B \leq 1.60$.

Diversos estudios han demostrado que las demandas sísmicas y el comportamiento estructural son particularmente sensibles a la relación entre el periodo fundamental de la estructura y el periodo predominante del terreno (Miranda 1994), de manera que aunque la inecuación propuesta es sólo función de la estructura, el periodo dominante del suelo se incluye en la determinación del factor de reducción para fines de diseño $Q$ ' como se establece en las NTCS-RCDF-04.

$$
S i\left\{\begin{array}{cr}
\frac{H}{B} \leq 1 & Q=3 \\
1<\frac{H}{B} \leq 1.6 & Q=3-\frac{5}{3}\left(\frac{H}{B}-1\right) \\
1.6<\frac{H}{B} & Q=2
\end{array}\right.
$$

En la figura 16 se ejemplifica el comportamiento de reducción por ductilidad con fines de diseño para marcos dúctiles con contraventeo concéntrico en función de la altura y la carga sísmica relativa $(1 / Q$ ') para evidenciar la dependencia de la fuerza sísmica de diseño de este factor de modificación considerando los razonamientos de algunos reglamentos internacionales considerando una base de $B=35$ m. Esto es, $Q^{\prime}$ para el NTCS-RCDF-04 y para el Manual de Obras Civiles de la Comisión Federal de Electricidad (MOC-CFE-08), $C_{d}$ en los más recientes de los Estados Unidos como el ASCE-7-05 e IBC$06, q$ en el reglamento europeo (EC8-05), $1 / D_{s}$ para el reglamento japonés (BCJ-06), $R_{d}$ en el reglamento canadiense (CNBC-05), suponiendo que el factor de importancia es $I=1.0$ cuando fue necesario. Los criterios que establecen cada código se discuten con mayor detalle en Tapia y Tena (2010). 


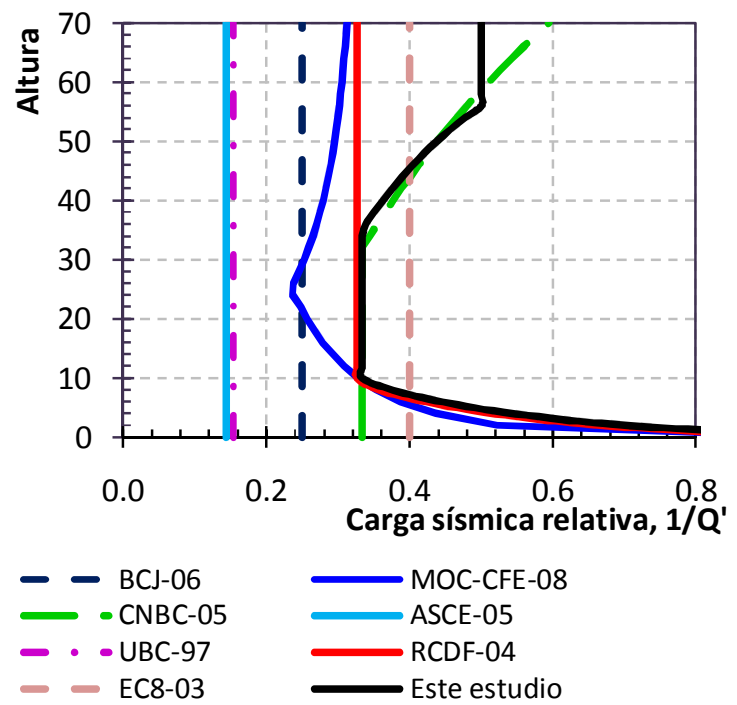

Figura 16. Carga mínima relativa con fines de diseño entre algunos reglamentos

La figura 16 incluye el comportamiento del factor de reducción con fines de diseño $Q$ ', obtenido con la aplicación de la metodología propuesta para la determinación de $Q$, considerando el procedimiento de las NTCS-04. Así, el límite aquí propuesto reconoce que en estructuras de mediana a gran altura con contravientos convencionales es necesario aumentar la carga sísmica de diseño para alcanzar las deformaciones inelásticas pretendidas. La propuesta presentada en este estudio coincide con el planteamiento de las NTCS-04 en modelos de baja altura cuando $H / B<1.0$, ya que ambas coinciden en establecer una magnitud máxima igual a $Q=3$.

En la figura 17 se compara el factor de reducción $Q$ con los límites propuestos por los reglamentos mexicanos (NTCS-RCDF-04 y MOC-CFE-08) y los obtenidos en los modelos estudiados en esta investigación. La metodología propuesta admite una disminución del factor de reducción siguiendo la tendencia de los resultados obtenidos en este estudio y coincide con la propuesta de los reglamentos mexicanos para relaciones de esbeltez de los edificios $H / B$ menores a 1.0.

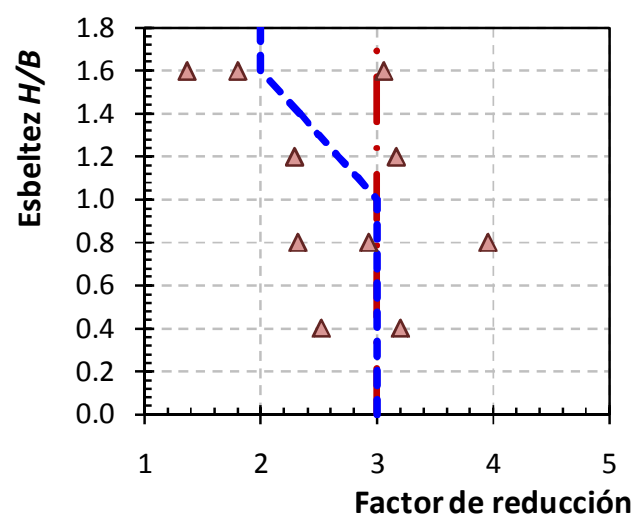

\footnotetext{
$\triangle \quad$ Curva primaria idealizada

- NTCS-04 y MOC-CFE-08
}

Figura 17. Comparación de las ductilidades de los modelos y los reglamentos mexicanos 


\section{FACTOR DE REDUCCIÓN POR SOBRERRESISTENCIA $(\Omega, R)$}

Además de la reducción debida a comportamiento inelástico, los reglamentos consideran otras reducciones para tomar en cuenta que la resistencia lateral real de las estructuras es mayor a la resistencia lateral de diseño. El considerar en forma explícita esta sobrerresistencia en los diseño, permite tener una mayor certidumbre del nivel de fuerzas laterales que puede actuar sobre las estructuras (Miranda 1994).

La tendencia actual para considerar el efecto de sobrerresistencia es reducir la carga de diseño por un factor de sobrerresistencia ( $R$ en los reglamentos mexicanos, $\Omega$ en los reglamentos de Estados Unidos) en lugar de incrementar la resistencia factorizada (Tena-Colunga et al. 2009). Las estructuras ven afectada su sobrerresistencia por varias fuentes: elección de las secciones estructurales que deben sujetarse a las existencias comerciales, tipificación de secciones un cierto número de pisos por razones constructivas, relación entre la resistencia nominal y factorizada, esfuerzo de fluencia real, endurecimiento por deformación y formación del mecanismo de colapso (Mitchell et al. 2003).

En la figura 18 se presenta las relaciones de esfuerzo obtenidas en la etapa elástica de diseño del modelo Ch16p8, por nivel distinguiendo la solicitación entre a) columnas, b) trabes y c) contravientos. Se observó una estrecha relación entre la sobrerresistencia y la contribución lateral al cortante resistente que soportan las columnas. Además, se aprecia un sobrediseño de las columnas a medida que resisten una mayor proporción del cortante lateral; algo similar se observa en las trabes y, en contraste, el diseño de los contravientos es cada vez más justo (menos sobrerresistencia) a medida que se diseñan para aportar menos cortante lateral. Este hecho redunda en que las columnas pueden desarrollar una alta sobrerresistencia, que se asocia a la concentración inminente del daño en los contravientos (mecanismo columna fuerte - viga débil - contraviento más débil).

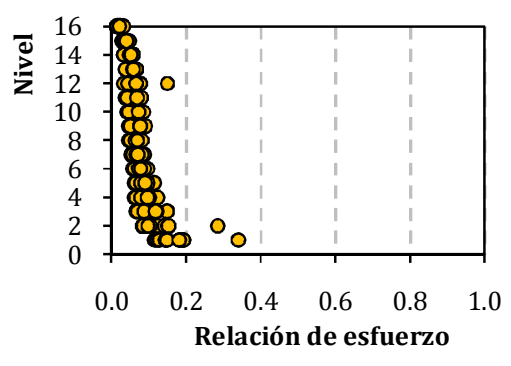

a) Columnas

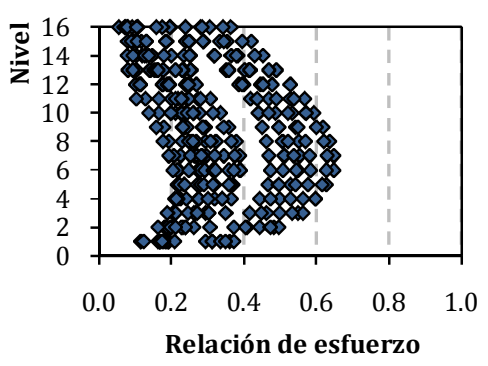

b) Trabes

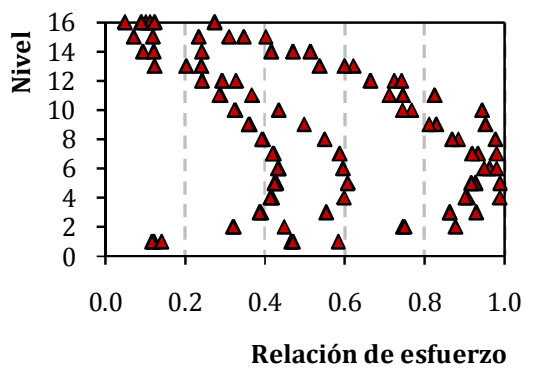

c) Contravientos

Figura 18. Relación de esfuerzos del modelo Ch16p8

Cabe señalar que aunque los contravientos se diseñaron para que su sección fuera lo más justa posible para el elemento crítico de un entrepiso dado, la sobrerresistencia que se observa en la figura 18 se debe a la práctica de tipificar secciones un determinado números de niveles, que tiene razones de peso desde el punto de vista constructivo y práctico que ya se enunciaron con anterioridad.

Estos resultados advierten las desventajas de añadir sobrerresistencia a los contravientos inconscientemente al tipificar secciones tal vez en exceso, o mediante las ayudas de diseño de los programas de análisis y diseño comerciales. Los contravientos sobrediseñados disipan menos energía y podrían concentrar el daño en las trabes y columnas lo que contribuye a mecanismos de colapso indeseables. En Tapia y Tena (2010a) se presenta una amplia discusión sobre las fuentes que proporcionan una sobrerresistencia en estructuras metálicas. 


\section{Discusión de resultados}

En la tabla 5 se reportan las sobrerresistencias $\Omega$ (cortante nominal $V_{\text {nom }}$ y el cortante máximo $V_{\text {max }}$ ) alcanzadas por los modelos estudiados considerando la curva global. La tabla incluye la magnitud del cortante basal nominal $V_{\text {nom }}$ mediante el que se realizaron los análisis ante carga monótona creciente y el cortante de fluencia medido al inicio del comportamiento inelástico $V_{y}$.

Tabla 5. Sobrerresistencias promedio obtenidas en los modelos estudiados

\begin{tabular}{cccccc}
\hline Modelo & \multirow{2}{*}{$V_{\text {basal }}$} & $V_{y}$ & $V_{\max }$ & $V_{\max } / V_{\text {nom }}$ & $V_{\max } / V_{y}$ \\
\hline Ch4p5 & \multirow{2}{*}{77.37} & 247.55 & 404.52 & 5.228 & 1.634 \\
Ch4p6 & & 381.64 & 442.68 & 5.722 & 1.160 \\
Ch8p5 & & 365.27 & 551.09 & 3.420 & 1.509 \\
Ch8p6 & \multirow{2}{*}{161.15} & 397.50 & 605.52 & 3.758 & 1.523 \\
Ch8p7 & & 569.39 & 919.90 & 5.708 & 1.616 \\
Ch12p5 & & 930.77 & 1177.35 & 4.807 & 1.265 \\
Ch12p6 & \multirow{2}{*}{44.95} & 1028.74 & 1302.02 & 5.315 & 1.266 \\
Ch12p8 & & 947.10 & 1438.39 & 5.872 & 1.519 \\
Ch16p5 & & 854.69 & 1809.42 & 5.504 & 2.117 \\
Ch16p6 & \multirow{2}{*}{328.73} & 1030.02 & 1284.05 & 3.906 & 1.247 \\
Ch16p8 & & 964.27 & 1515.68 & 4.611 & 1.572 \\
\hline
\end{tabular}

En la figura 19 se grafican las sobrerresistencias obtenidas contra el número de niveles de los modelos distinguiendo el porcentaje de aportación al cortante lateral resistente, mientras que en la figura 20 se presenta el comportamiento de la proporción del cortante máximo alcanzado en los análisis $V_{\max } \mathrm{y}$ el cortante de fluencia $V_{y}$ de las curvas cortante - distorsión, para hacer notar que la fluencia de los entrepisos se presenta a magnitudes mayores que el cortante nominal $V_{\text {basal }}$ con el que se realizaron los análisis estáticos no lineales.

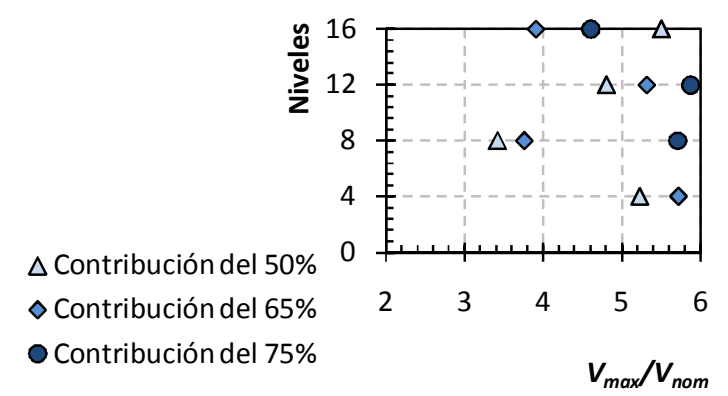

Figura 19. Comportamiento de la sobrerresistencia $\Omega$ en función de la altura

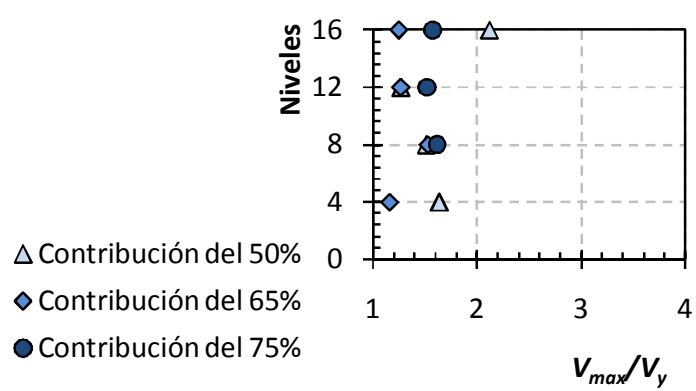

Figura 20. Comportamiento de la proporción $\mathrm{V}_{\max } / \mathrm{V}$ en función de la altura de los modelos 
Las sobrerresistencias globales alcanzadas por los modelos son siempre mayores a los valores indicativos propuestos en los reglamentos en estudio, sin que se observe alguna dependencia con la altura o con la contribución del cortante lateral resistente aportado para el que fueron diseñadas las columnas de los modelos. Las gráficas no incluyen los resultados de los modelos que no cumplen la aportación mínima del $50 \%$ al cortante lateral resistente, debido a que presentaron una falla frágil.

Estos resultados concuerdan con los obtenidos en estudios previos (Tapia 2005, Tapia y Tena 2009), donde se reportan sobrerresistencias para estos mismos sistemas estructurales iguales a $\Omega=4.72 \mathrm{y}$ $\Omega=4.55$ en modelos de 15 niveles, lo que sugiere que las magnitudes de los factores por sobrerresistencia establecidos en los reglamentos no son representativas de la sobrerresistencia que pueden desarrollarse en estos sistemas estructurales si se diseñan conforme a esos mismos reglamentos, pese a que la magnitud de este factor varía ampliamente en función de la configuración y el criterio de diseño sísmico.

\section{Tendencias de reglamentos vigentes}

Conforme a la sección 11.2.1 del documento ATC 63 (2008), los factores por sobrerresistencia de la tabla 12.2-1 del reglamento norteamericano ASCE/SEI 7-05 (2005), que varían entre $\Omega=2$ y $\Omega=3$, no concuerdan con los valores calculados en investigaciones recientes que varían entre $\Omega=1.5$ (en el peor de los casos) hasta más de $\Omega=6.0$.

El reglamento canadiense propone sobrerresistencia para marcos de acero con contraventeo concéntrico iguales $\Omega=1.3$ cuando se trata de marcos de ductilidad limitada, y $\Omega=1.5$ en marcos dúctiles, asociados a ductilidades iguales a $\mu=2.0$ y $\mu=3.0$, respectivamente (CNBC-05 2005).

El reglamento del Distrito Federal presenta en el Apéndice A (NTCS-RCDF-04) una ecuación que tiende a un límite inferior $\Omega=2$, sin que haga diferencia del sistema estructural, a diferencia de lo que se propone en versiones recientes de reglamentos como ASCE 7-2005, IBC-2006 y MOC-CFE-08, donde se proponen valores constantes de $\Omega$ que dependen del sistema estructural (Tena-Colunga et al. 2009). El planteamiento del Apéndice A de las NTCS-RCDF-04 es función del periodo característico $T_{a}$ del espectro cuya definición es igualmente función del terreno del suelo, conforme se ilustra en la figura $21\left(T_{a}=1.175\right.$ $\mathrm{s}$, cuando el periodo fundamental del suelo es $T_{a}=2 \mathrm{~s}$ ). La figura incluye el segundo periodo característico $T_{b}$ que define el descenso de las aceleraciones máximas.

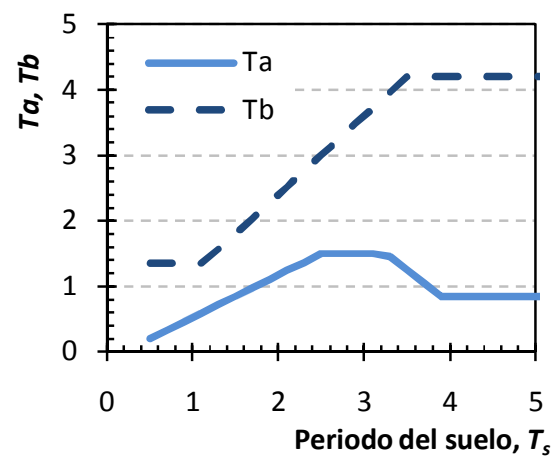

Figura 21. Comportamiento de periodos característicos según Apéndice A

El comportamiento de la propuesta de las Normas Técnicas se ilustra en la figura 22, para un periodo fundamental del terreno igual a $T_{s}=2 \mathrm{seg}$. En la misma figura, se han incluido las sobrerresistencias obtenidas en este estudio en función del periodo fundamental de los modelos y 
distinguiendo el porcentaje de aportación al cortante lateral resistente para el que se diseñaron las columnas.

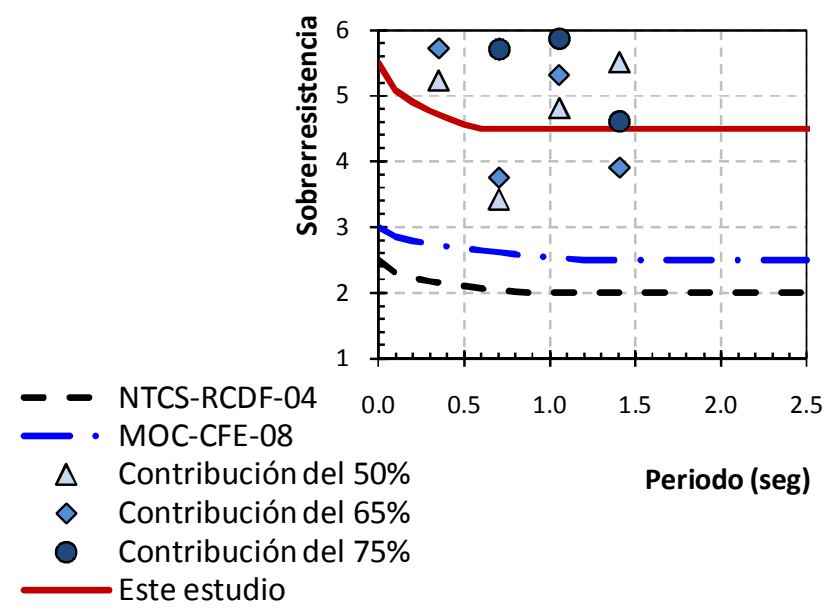

Figura 22. Comportamiento de las sobrerresistencias contra lo estipulado en reglamentos mexicanos

Por otra parte, el Manual de Obras Civiles de la Comisión Federal de Electricidad (Tena-Colunga et al. 2009) presenta un método de obtención del factor por sobrerresistencia que depende de un índice de sobrerresistencia $R_{0}$ para considerar el sistema estructural (ecuación 4).

$$
R=\left\{\begin{array}{lr}
R_{0}+a\left(1-\sqrt{\frac{T}{T_{a}}}\right) & \text { si } T \leq T_{a} \\
R_{0} & \text { si } T>T_{a}
\end{array}\right.
$$

Se propone $\alpha=0.5$ para todos los sistemas y $R_{0}=2.0$ para marcos momento resistentes de ductilidad normal $(Q=2)$ e intermedia $(Q=3)$, marcos momento resistentes de ductilidad normal con contraventeo $(Q=2)$ y para muros de mampostería con piezas de sección hueca; $R_{0}=2.5$ para-marcos momento resistentes de ductilidad alta $(Q=4)$, marcos contraventeados dúctiles diseñados con $Q=3$ y muros de mampostería confinada con piezas sólidas; $R_{0}=3.0$ para estructuraciones mixtas dúctiles diseñadas con $Q=4$, como marcos contraventeados o con muros de concreto reforzado o de placa de acero o compuestos de los dos materiales. El comportamiento de este criterio se incluye en la figura 22 con un $R_{0}=2.5$ para marcos de acero dúctiles con contraventeo y un periodo característico $T_{a}=1.175 \mathrm{seg}$.

A fin de establecer una propuesta conservadora para estimar la sobrerresistencia en forma realista en marcos de acero con contravientos concéntricos, se adaptaron las ecuaciones propuestas en el Apéndice A de las NTCS-RCDF-04 y del MOC-CFE-08 con $R_{0}=4.5$ y $\alpha=1.0$ (ecuación 4), utilizando la curva media aproximada de los resultados obtenidos, a partir de las magnitudes mínimas reportadas en la tabla 5.

El límite propuesto en esta investigación (ecuación 4) reconoce que estructuras de baja altura podrían estar asociados a mayores sobrerresistencias, de hasta $\Omega=5.5$ y mayores ductilidades (en congruencia al criterio propuesto en el apartado anterior) y establece una sobrerresistencia igual a $\Omega=4.5$, cuando el periodo de la estructura supere al periodo característico $T_{a}$. En la figura 22 se ejemplifica el comportamiento de la propuesta, que genera magnitudes de sobrerresistencia menos conservadoras y mucho más aproximados a los resultados obtenidos en ésta y otras investigaciones. 
La metodología propuesta es función del periodo de la estructura, reconociendo que tanto la sobrerresistencia como la reducción por comportamiento no lineal presentan importantes variaciones de las demandas de ductilidad global dependiendo del periodo dominante del edificio (Miranda 1994).

\section{CONCLUSIONES Y RECOMENDACIONES}

Este artículo presenta los resultados de análisis estáticos ante carga monótona creciente de 13 edificios regulares de acero con marcos dúctiles con contraventeo concéntrico. Los edificios se supusieron ubicados en suelo blando conforme al Reglamento de Construcciones para el Distrito Federal (RCDF-04), que son similares a otros reglamentos internacionales (CNBC, LRFD-AISC, ASCE, IBC, EuroCode). Los modelos estudiados tienen alturas entre cuatro y 16 niveles con dos diferentes configuraciones de contravientos. Los edificios fueron diseñados con diferentes contribuciones al cortante lateral resistente contribuido por las columnas del marco en relación al sistema de contravientos.

Los análisis obtenidos sugieren que el mecanismo de colapso supuesto en la etapa de diseño (columna fuerte - viga débil - contraviento más débil) no se obtiene en todos los casos siguiendo el planteamiento propuesto en el RCDF-04 en estructuras ubicadas en terreno blando. Este estudio evidencia una estrecha relación entre el mecanismo desarrollado con la altura o relación de esbeltez del edificio no considerada explícitamente en los reglamentos vigentes.

Con los resultados obtenidos se propuso una expresión para definir una contribución mínima al cortante lateral resistente que deben aportar las columnas del marco en relación al sistema de contravientos para obtener un mecanismo de colapso consistente con las hipótesis de diseño. Esta expresión toma en cuenta la relación de esbeltez del edificio y el balance de esfuerzos de fluencia de los elementos estructurales.

Se demostró que el límite de deformación por fluencia (revisión del estado límite de servicio) propuesto en el RCDF-04 es bastante aproximado a los obtenidos de los análisis no lineales. Sin embargo, el análisis de las distorsiones últimas promedio obtenidas, $\delta_{u}$, sugiere una revisión de la distorsión límite establecida en las Normas Técnicas para la revisión del estado de prevención de colapso, debido a que en la mayoría de los modelos ésta resultó menor a $\delta_{u-p e r m}=0.015$. Para ello sería recomendable realizar experimentos a escala natural en edificios representativos con base en marcos contraventeados de varias crujías y varios niveles, los que pudieran llevarse a cabo en el laboratorio E-Defense de Kobe, Japón.

Se demostró que el factor de reducción debido al comportamiento no lineal de la estructura tiene una marcada dependencia con la esbeltez de los modelos (ancho contra altura), no considerada en el reglamento vigente y que coincide con otras investigaciones relacionadas (por ejemplo, Miranda 1994), donde se ilustra la alta dependencia de las demandas de ductilidad con el periodo fundamental de la edificación.

Cuando los modelos satisfacen el límite de aportación mínima al cortante resistente del cincuenta por ciento, las estructuras de baja o mediana altura satisfacen las ductilidades pretendidas en la etapa de diseño $(Q=3)$. Este comportamiento favorable se deteriora con el aumento de la altura, de tal manera que se presentó una metodología para definir un factor de reducción por ductilidad en función de la relación de esbeltez, a fin de proporcionar una mayor certidumbre para poder controlar la demanda de ductilidad global en estos sistemas estructurales. El método propuesto es congruente con los resultados de ésta y otras investigaciones. 
En todos los casos, las sobrerresistencias obtenidas en los análisis resultaron ser mayores a lo especificado en los reglamentos considerados en este estudio (NTCS-RCDF-04, MOC-CFE-08, CNBC05, ASCE-7-05 e IBC-06), sin que se notara una dependencia de la aportación al cortante lateral resistente para el que fueron diseñadas las columnas ni de la altura de los modelos. A partir de los resultados, se propuso una adecuación de la metodología presentada en el Apéndice A de las NTCS-RCDF-04 y en el MOC-CFE-08 proponiendo una sobrerresistencia máxima igual a $R=5.5$ en modelos de baja y mediana altura disminuyendo hasta $R=4.5$ con el aumento de la altura.

En este trabajo se supuso que las conexiones de los marcos momento - resistentes eran rígidas, por lo que los resultados se deben considerar con las reservas necesarias en marcos con conexiones semirígidas o flexibles.

\section{AGRADECIMIENTOS}

El primer autor del artículo desea agradecer a la Comisión Nacional de Ciencia y Tecnología (Conacyt) su apoyo para la realización de este estudio que forma parte de su investigación doctoral.

\section{REFERENCIAS}

ASCE 7-05 (2005), "Minimum design loads for buildings and other structures", ASCE Standard ACE/SEI 7-05, American Society of Civil Engineers, ISBN 0-7844-0809-2.

ATC - 63 (2008), Quantification of buildings seismic performance factors, ATC-63 Project Report-90\% Draft. FEMA P695, abril.

Bruneau, M, C-M Uang y A Whittaker (1998), Ductile design of steel structures, Mc Graw Hill, Boston, Massachussetts.

CNBC-05 (2005), "Code National du Bâtiment-Canada 2005. Volume 1", Commission canadienne des codes du bâtiment et de prévention des incendies. Conseil National de Recherché, doceava edición, Ottawa, Canadá.

Elghazouli, A (2003), "Seismic design procedures for concentrically braced frames", Structures and Buildings, Vol. 156, No. SB4, noviembre.

Fell, B V, A M Kanvinde y G G Deierlein (2010), "Large-scale testing and simulation of earthquake induced ultra low cycle fatigue in bracing members subjected to cyclic inelastic buckling", Blume Earthquake Engineering Center Technical Report \#172, Stanford University, Stanford, CA.

Izvernari, C (2007), "The seismic behavior of steel braces with large sections", Mémoire du diplôme de maîtresse ès sciences appliqués Génie Civil, Département des Génies Civil, Géologique et des Mines, École Polytechnique de Montréal, Canadá, abril.

Kathib, I, S Mahin y K Pister (1998), "Seismic behavior of concentrically braced steel frames", Report UBC/EERC-88/01, Earthquake Engineering Research Center, University of California, enero.

Kemp, R A (1996), "Inelastic local and lateral buckling in design codes", ASCE Journal of Structural Engineering, Vol. 122, No. 4, pp. 374-382, abril.

Lacerte, M y R Tremblay (2006), "Making use of brace overstrength to improve the seismic response of multistory split-X concentrically braced steel frames", Canadian Journal of Civil Engineering, Vol. 33, pp. 1005-1021. 
MacRae, G A, Y Kimura y C Roeder (2004), "Effect of column stiffness on braced frame seismic behavior", Journal of Structural Engineering, ASCE, Vol. 130, No. 3, pp. 381-391, marzo.

Miranda, E (1994), "Observaciones a los criterios de diseño sismorresistente del reglamento de construcciones para el Distrito Federal”. Revista de Ingeniería Sísmica. No. 50, p.p. 15-30, septiembre.

Mitchel, D, R Tremblay, E Karacabeyli, P Paultre, M Saatcioglu y D Anderson (2003), "Seismic force modification factors for the proposed 2005 edition of the National Building Code of Canada", Canadian Journal of Civil Engineering, Vol. 30, pp. 308-327.

Prakash, V, G H Powell y F Fillipou (1992), "Drain-2DX: Base Program User Guide”, Report No. $U C B / S E M M-92 / 29$, Department of Civil Engineering, University of California at Berkeley.

RCDF-04 (2004), "Reglamento de Construcciones para el Distrito Federal (RCDF)", Gaceta Oficial del Distrito Federal, décima cuarta época, enero.

Remennikov, A M y W R Walpole (1998). "Seismic behavior and deterministic design procedures for steel V-braced frames", Earthquake Spectra, Vol. 14, No. 2, pp 335-355, mayo.

Tapia, E (2005), "Estudio de los criterios del RCDF-2004 y sus Normas Técnicas Complementarias para el diseño sísmico de edificios regulares con base en marcos de acero", Tesis de Maestría, División de Estudios de Posgrado de la Facultad de Ingeniería, Universidad Autónoma de México, abril.

Tapia, E y A Tena-Colunga (2008), "Behavior of regular steel moment resisting concentrically braced frames (MRCBFs) in seismic zones", Memorias, $14^{\text {th }}$ World Conference on Earthquake Engineering, Beijing, China, Artículo No. 05-05-0008, CD-ROM, octubre.

Tapia, E y A Tena (2009), “Comportamiento sísmico de edificios regulares con marcos dúctiles de acero con contraventeo concéntrico diseñado conforme al Reglamento del Distrito Federal mexicano", Revista Internacional de Ingeniería en Estructuras, Vol. 13 y 14, No. 1, pp. 1-28.

Tapia, E y A Tena (2010), "Estudio del comparativo de las previsiones sísmicas de edificios estructurados con marcos de acero", Memorias del XVII Congreso Nacional de Ingeniería Estructural. León, Guanajuato.

Tapia, E y A Tena (2010a), “Observaciones sobre criterios de diseño sísmico de edificios con marcos de acero”, Revista Internacional de Ingeniería en Estructuras, Vol. 15, No. 2, pp. 157-180.

Tapia, E (2011), "Estudio del comportamiento de edificios regulares estructurados con marcos dúctiles de acero con contraventeo concéntrico en suelos blandos", Tesis de Doctorado, Posgrado en Ingeniería Estructural, División de Ciencias Básicas e Ingeniería, Universidad Autónoma Metropolitana Azcapotzalco (en prensa).

Tena-Colunga, A, U Mena-Hernández, L E Pérez-Rocha, J Avilés, M Ordaz y J I Vilar (2009), “Updated seismic design guidelines for buildings of a model code of Mexico", Earthquake Spectra, Vol. 25, No. 4, pp. 869-898, noviembre, doi: 10.1193/1.3240413. 